\title{
Auf dem Wege zum grätenlosen Karpfen*
}

\author{
R. v. SENGbusCh und CH. MESKE
}

Max-Planck-Institut für Kulturpflanzenzüchtung, Hamburg-Volksdorf

\section{On the Way to Boneless Carp}

Summary. The examination of about 2.000 carps of one population by röntgenograms has shown a large diversity of the number of intermuscle bones. With an average of about 100 bone tips per fish, the extremes were 70 and 134 bone tips per fish. It was ascertained, that the number of bone tips increases during the first 2 years of life, whereas the number of bone bases does not change. Due to the law of parallel mutation, we expect to find types with extremely few or without bones.

Die Qualität einer großen Zahl von Süßwasserfischarten wird entschieden durch die Anzahl der Zwischenmuskelgräten ungünstig beeinflußt.

Diese außerhalb des Skelettsystems liegenden Bindegewebsverknöcherungen treten bei einigen Seefischarten kaum oder überhaupt nicht auf, z. B. beim Kabeljau (Gadus morruha), bei dem die Zwischenmuskelgräten fehlen (vgl. die Abbildungen bei v. Sengbusch 1963). Es kann daher angenommen werden, daß die Zwischenmuskelgräten zur Stabilisation des Fischkörpers nicht notwendig sind.

Auf Grund der Gesetze der Parallelvariation bzw. -mutation kann man auf die Existenz von grätenlosen Exemplaren auch bei an sich grätenreichen Fischarten schließen. Diese Annahme wird auch durch die unterschiedliche Anzahl der Zwischenmuskelgräten unterstützt, die bei den verschiedenen Süßwasserfischarten auftreten.

LIEDER '(1961) untersuchte 17 Arten von einheimischen Süßwasserfischen und stellte Grätenzahlen von 21 (Kaulbarsch) bis 143 (Rapfen) fest.

* Herrn Professor Dr. Hans StubBe zum 65. Geburtstag gewidmet.
Als Methode zur Bestimmung der Grätenzahl hat sich die Anfertigung von Röntgenaufnahmen bewährt. Es konnte gezeigt werden, daß bereits bei jungen Karpfen von 7,5 cm Länge die Zwischenmuskelgräten deutlich zu erkennen sind (v. SENGBUSCH 1963).

Als ,,schnell-genug-Methode“ ist die Anwendung der Röntgenphotographie jedoch nicht optimal, da zur Auslese grätenloser Mutanten wahrscheinlich eine sehr große Anzahl von Karpfen untersucht werden muß. Hierfür erscheint die direkte Sichtauslese geeigneter. Mit Hilfe der bisher üblichen Schirmbildgeräte waren jedoch die $Z$ wischenmuskelgräten nicht sichtbar zu machen.

Die züchterische Bearbeitung unserer Süßwassernutzfische - also auch des Karpfens - im Hinblick auf ihre Grätenzahl ist bisher noch nicht vorgenommen worden. Wenngleich im Laufe der Jahrhunderte verschiedene Körperformen der Karpfen gezüchtet worden sind, die sich in erster Linie in Höhen-Längen-Quotienten unterscheiden (Galizische, Böhmische, Aischgründer Rasse), und wenngleich man auch verschiedene Beschuppungstypen des Karpfens durch systematische Zuchtwahl erreicht hat (z. B. Spiegelkarpfen, Nacktkarpfen), so ist doch bisher jeder Versuch unterblieben, die Anzahl der Zwischenmuskelgräten durch züchterische Maßnahmen zu reduzieren.

$\mathrm{Da}$ anzunehmen ist, $\mathrm{daB}$ der Konsum solch schmackhafter Süßwasserfische, wie der Karpfen, durch Grätenlosigkeit in hohem Maße zunehmen wird - besonders Familien mit Kindern meiden den Genuß von Süßwasserfischen --, setzten wir uns das Ziel, die züchterische Bearbeitung dieses Problems in Angriff zu nehmen (vgl. v. Sengbusch 1963). 
Der erste Schritt hierzu mußte in der Feststellung der vorhandenen Variation der Grätenanzahl liegen. LIEDER hatte in seiner erwähnten Arbeit lediglich elf Karpfen untersucht. Weitere Angaben liegen bisher im Schrifttum nicht vor. Die vorliegende Arbeit gibt eine Übersicht über unsere ersten Untersuchungen auf diesem Gebiet.

Parallel zu den Arbeiten über die Zwischenmuskelgräten hielten wir zur Durchführung der geplanten züchterischen Arbeiten die kontrollierbare Aufzucht der Karpfen unter Laboratoriumsbedingungen für unerläßlich.

Wir entwickelten daher eine neuartige Methode der Fischhaltung, die es ermöglicht, die Aufzucht von Karpfen vom Ei bis zum geschlechtsreifen Fisch unter Laboratoriumsbedingungen durchzuführen. Den sogenannten Raumfaktor konnten wir durch ständigen Wasserdurchfluß durch die Aquarien ausschalten. Durch Einbau einer biologischen Wasserklärung konnten wir einen geschlossenen Wasserkreislauf konstruieren, der die Einhaltung einer konstanten Temperatur ermöglicht und auf engstem Raum einen ganzjährigen Zuwachs der Karpfen ergibt (v. SENGBUSCH et al. 1965, 1966, 1967).

Bei Anwendung dieser Verfahren konnten wir bereits bei zweijährigen Karpfen die Geschlechtsreife erzielen. Durch Injektion von Karpfen-Hypophysen-Material erreichten wir bei diesen Tieren die Abgabe der Geschlechtsprodukte. Nach der Methode von WoYNÁRovich (1961) wurde die Behandlung des Laiches durchgeführt, der dann in Zugergläsern bis zum Schlüpfen der Brut unter ständigem Wasserdurchfluß gehalten wurde. Die Gewinnung und Aufzucht von Karpfenbrut gelang uns unabhängig von der Jahreszeit, bisher im August, im Januar, Februar und März. Beachtlich ist, daß die gleichen Elterntiere im August und im darauf folgenden Januar vollwertigen Laich absetzten (vgl. Meske 1966, MEske et al. 1967).

Die Aufzucht der Brutfische gelang im Aquarium in den ersten Lebenswochen mit Trockenfutter unter Zugabe von Protozoen und später Artemia-Larven.

Für die züchterische Bearbeitung von Fischen eröffnen sich durch diese Ergebnisse völlig neue Möglichkeiten.

\section{Material und Methode}

Die Karpfen, die wir auf die Anzahl ihrer Zwischenmuskelgräten hin untersuchten, stammten alle aus einer Teichwirtschaft und sind nach Angaben des Teichwirtes seit vielen Generationen rein weitervermehrt worden. Untersucht wurden insgesamt ca. 2000 Karpfen.

Ein Teil der Tiere wurde lebend geröntgt, nachdem sie mit Urethan (1:100) bzw. später mit MS 222 (SANDOZ) (1:50000) narkotisiert worden waren.

Der größte Teil der Karpfen bestand aus toten Tieren, die bei der Frühjahrsabfischung angefallen waren. Die Aufnahmen wurden mit Hilfe eines Röntgenapparates Typ Müller MG 150 durchgeführt. Die Aufnahmedauer stand in Abhängigkeit von der Länge des Fisches. Die Mehrzahl der Tiere wurde mit $70 \mathrm{kV}$ bestrahlt (Karpfen von ca. 15-20 cm Länge). Als Filmmaterial verwendeten wir Gevaert Röntgenfilm Structurix D4, entwickelt wurde in Agfa Radional-PRöntgen-Rapid-Entwickler. Die Auswertung der
Filmbilder erfolgte unter einer binokularen Lupe bei 7 facher Vergrößerung mit Durchlichtbeleuchtung durch Leuchtstoffröhren.

Neben den ausgezählten Gräten der Röntgenphotos wurden 14 Karpfen nach dem Röntgen leicht angekocht und die $Z$ wischenmuskelgräten herauspräpariert. Diese Kontrolluntersuchungen deckten sich weitestgehend mit den Befunden aus den Röntgenbildern.

Beim Auszählen der Zwischenmuskelgräten mußten wir eine Unterscheidung treffen in Anzahl der Grätenspitzen und Anzahl der Grätenbasen. Im ersten Fall ist also z. B. bei einfach gegabelten Elementen zwei, im zweiten Fall eins zu zählen. Um Zählfehler weitgehend zu vermeiden, etwa durch das Verwechseln zweier sich kreuzender unverzweigter Gräten mit einer einfach gegabelten, zählten wir bei der Masse der Fische die Anzahl der Grätens pitzen.

\section{Ergebnisse}

Die Auswertung der Röntgenaufnahmen von $704 K_{1}$ (halbjährig) ergab eine beachtliche Streuung der Anzahl der Zwischenmuskelgrätenspitzen. Wie die $\mathrm{Abb}$. 1 veranschaulicht, traten Extremwerte von nur 70 Grätenspitzen pro Fisch auf. Andererseits waren maximal 134 Grätenspitzen festzustellen. Der Durchschnittswert aus dieser Gruppe von 704 Karpfen liegt bei 100 Grätenspitzen pro Fisch. Die beobachtete Variabilität der Grätenzahl ist deshalb besonders beachtlich, weil es sich, wie erwähnt, um gleichaltrige Tiere einer Zucht handelt.

Um eine Relation zwischen der Anzahl der Grätenspitzen zu der der Grätenbasen aufzustellen, zählten wir bei 100 willkürlich ausgelesenen Karpfen der oben beschriebenen Gruppe die Grätenbasenanzahl parallel zur Anzahl der Grätenspitzen aus. Naturgemäß lag der Durchschnittswert für Basen niedriger als der für Spitzen. Die Abb. 2 zeigt eine Gegenüberstellung der ermittelten Werte. Die 100 untersuchten halbjährigen Karpfen wiesen im Durchschnitt 100 Grätenspitzen (Extremwerte 70 und 134) und 77 Grätenbasen (Extremwerte 56 und 95) auf.

Bei Einzeluntersuchungen größerer Karpfen fiel uns die starke Aufspaltung der $Z$ wischenmuskelgräten auf, also die relativ hohe Anzahl von Grätenspitzen. Gräten mit 3, 4, 5 und mehr Spitzen waren weitaus häufiger zu beobachten als bei jüngeren Fischen. Wir untersuchten daher eine Gruppe von wei-

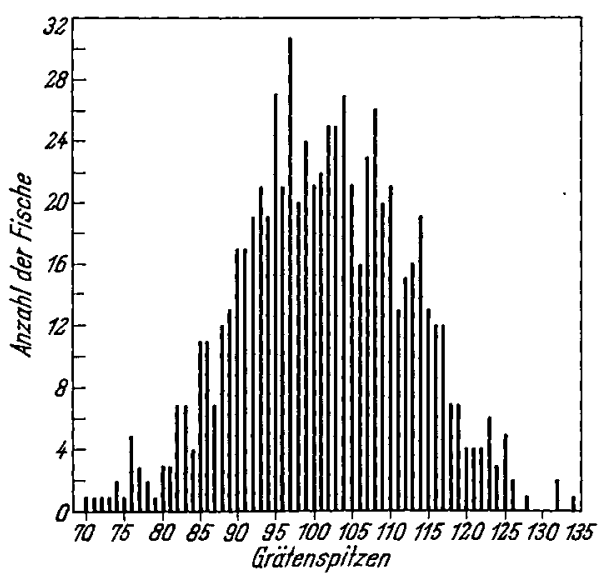

Abb. 1. Streuung der Anzabl der Grätenspitzen bei $70_{4}$ halbjährigen Karpfen einer Zucht. 


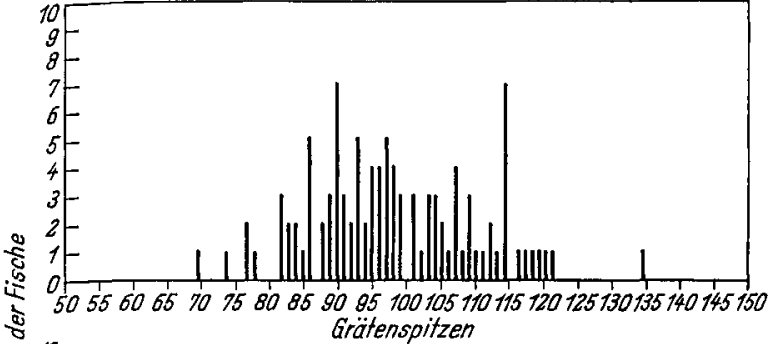

10

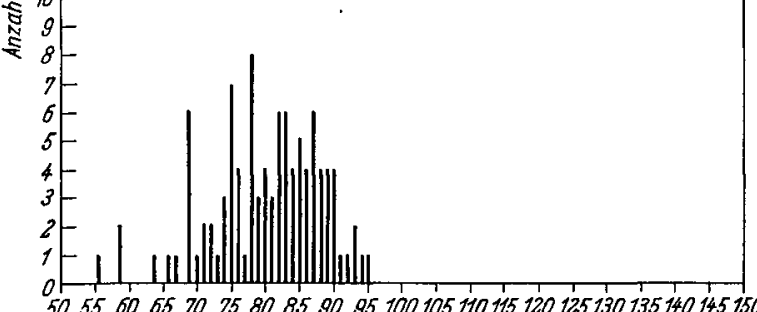

$505560 \quad 65707580858085100105110115120125130135140145150$ Gratenbasen

Abb. 2. Streuung der Anzahl der Grätenspitzen und Grätenbasen bei 100 halbjährigen Karpfen.

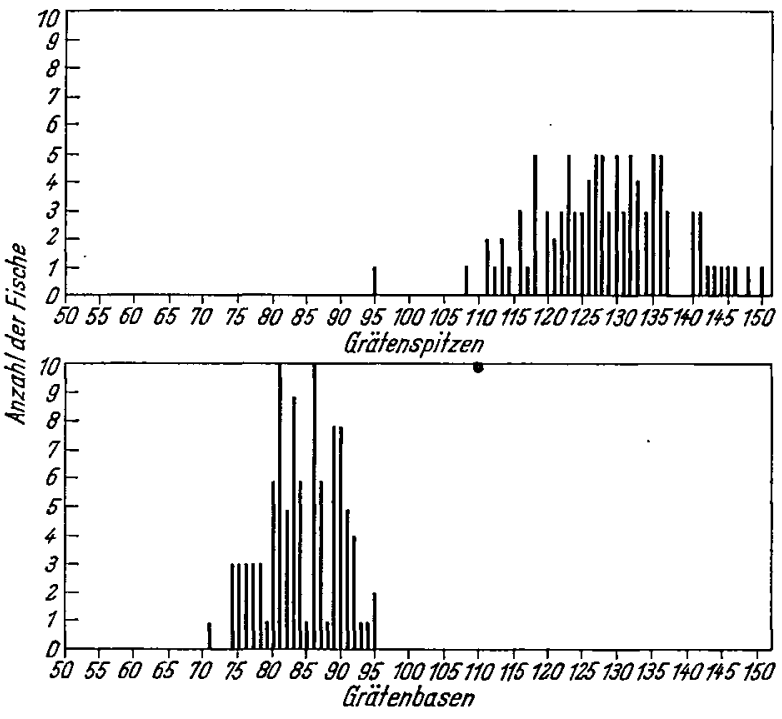

Abb. 3. Streuung der Anzahl der Grätenspitzen und Grätenbasen bei 100 eineinhalbjährigen Karpfen.

teren 100 Karpfen, die anderthalb Lebensjahre alt waren. Die Auszählung ergab eine deutliche Zunahme der Grätenspitzenzahl gegenüber den halbjährigen Karpfen. Auf der Abb. 3 macht die Gegenüberstellung von Spitzen- und Basenanzahl die Verschiebung der Grätenspitzenhäufigkeit nach rechts deutlich. Der Durchschnittswert beträgt hier 129 Grätenspitzen pro Fisch (Extremwerte 95 und 150) und 84 Grätenbasen (Extremwerte 71 und 95).

Die $1^{1} / 2$ jährigen Fische entstammten der gleichen Zucht wie die untersuchten halbjährigen Karpfen. Die erhebliche Zunahme der Grätenspitzenzahl bei den älteren Karpfen macht deutlich, daß bereits im Laufe eines Lebensjahres eine starke Aufspaltung der Zwischenmuskelgeräten erfolgt.

Die einmal angelegten Stämme der Basen der $Z$ wischenmuskelgräten scheinen in ihrer ursprünglichen Anzahl im Laufe der Ontogenese konstant zu bleiben.

Die Unterschiede in Grätenspitzen- und Grätenbasenanzahl sind - richtig belichtete Bilder vorausgesetzt - schon bei flüchtigem Betrachten der Photos auffallend. Auf der Abb. 4 ist ein Ausschnitt einer
Röntgenaufnahme eines $1 / 2$ jährigen Karpfens wiedergegeben, dessen Gesamtauszählung 104 Grätenspitzen und 92 Grätenbasen ergeben hatte.

Die Abb. 5 gibt dagegen einen entsprechenden Ausschnitt eines gleichaltrigen Karpfens wieder, der insgesamt nur 71 Grätenspitzen und 56 Grätenbasen aufwies. Betrachtet man beide Abbildungen im Vergleich, so ist die Differenz der Grätenanzahl bereits bei kurzer Betrachtung auffallend. Noch deutlicher kommt sie zum Ausdruck, wenn man die Gräten abzählt, die jeweils zwei Dornfortsätze ,kreuzen“.

In Abb. 4 sind dies z. B. 6 Zwischenmuskelgräten, in Abb. 5 dagegen nur 3.

Diese leicht erkenntlichen Unterschiede veranlaßten uns zu der Entwicklung einer Methode, die die Durchsicht einer größeren Anzahl von Karpfen in kurzer Zeit gestattet.

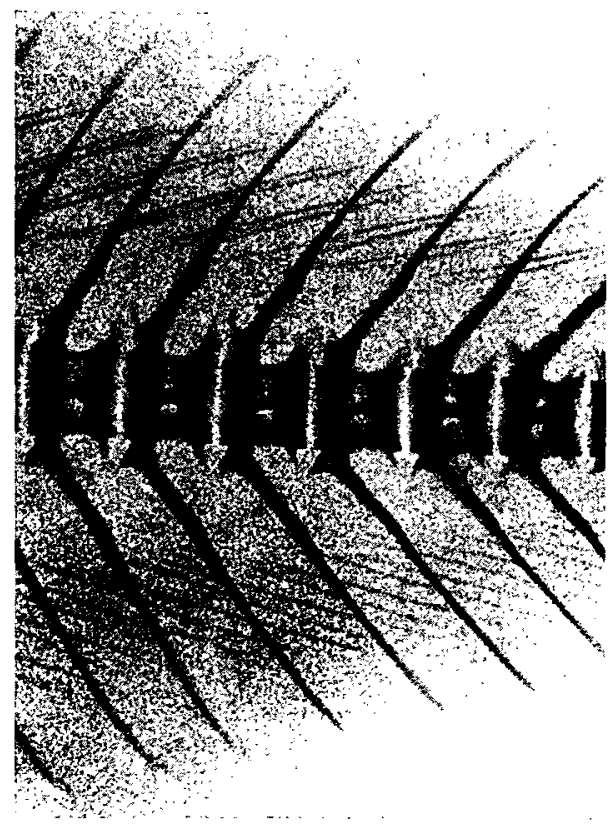

Abb. 4. Ausschnittsvergrößerung der Röntgenaufnahme eines halbjährigen Karpfens mitinsgesamt 92 Zwischenmuskelgräten.

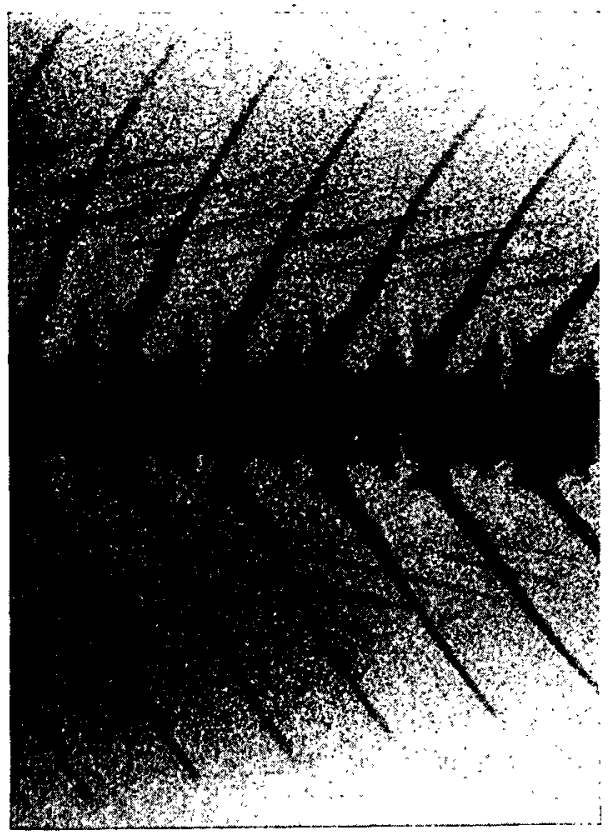

Abb. 5. Ausschnittsvergrößerung der Röntgenaufnahme eines halbjährigen Karnfens mit insgesamt; 56 Zwischenmuskelgräten. 
Hier werden nur die Schwanzwurzeln der Fische geröntgt. Aufgenommen wird auf Filmstreifen von $6 \times 48 \mathrm{~cm}$ Gevaert Strukturix D 2. Ein Bleistreifen, der in einer Führungsschiene läuft, ermöglicht die Aufteilung des Filmstreifens in zwei Längshälften. Auf diese Weise können bis zu 24 Fischschwänze auf einem $6 \times 48 \mathrm{~cm}$ Streifen aufgenommen werden, da der Schwanzflossensaum hierbei jeweils unter dem Bleistreifen liegt.

Auf diese Weise haben wir bisher ca. 1000 tote Karpfen untersucht, die bei Abfischungen im Frühjahr anfielen. Sie stammten alle aus der gleichen Population wie die untersuchten lebenden 704 Karpfen, über deren Grätenzahl oben berichtet wurde. Traten bei Auswertung der Ausschnittsbilder Extremwerte auf, so wurde der ganze Fisch noch einmal geröntgt und die Gräten in ihrer Gesamtheit ausgezählt.

Die Auswertung dieser Filmstreifen ergab eine Bestätigung der Variabilität der Grätenzahl bei der untersuchten Population. Extremere Werte als oben angegeben (70 bzw. 134 Grätenspitzen pro Fisch) traten jedoch nicht auf.

\section{Diskussion}

Wie eingangs erwähnt, ist anzunehmen, daß die Zwischenmuskelgräten der Karpfen keine stabilisa.torische Bedeutung für den Fischkörper haben. Für diese Annahme spricht das Vorhandensein extrem grätenarmer und auch völlig grätenloser Fischarten. Auch die relative Hochrückigkeit des Karpfens erfordert nicht das Vorhandensein der Zwischenmuskelgräten. Nach den Untersuchungen LIEDERs hat nämlich der ebenfalls relativ hochrückige Barsch (Perca fluviatilis) nur 21 Gräten im Durchschnitt, der sehr flachrückige Rapfen (Aspius aspius) dagegen 143 Gräten.

Die große Variabilität der Anzahl der Zwischenmuskelgräten bei den von uns untersuchten Karpfen unterstützt unsere Annahme, daß den Gräten funktionell keine Bedeutung zukommt. Die Verteilung der Grätenzahl, wie sie auf $\mathrm{Abb}$. 1 deutlich wird, entspricht in etwa der Gaußschen Fehlerkurve. Selbst bei einer erheblich größeren Anzahl von Fischen der gleichen Population wird sich an dieser Normalverteilung kaum etwas ändern. Bei anderen Populationen, deren Untersuchung folgen soll, kann eine ähnliche Kurve evtl. mit einer Mittelverschiebung nach links oder rechts erwartet werden. Durch Weiterzüchtung der grätenärmsten Exemplare läßt sich auf die Dauer wahrscheinlich die Anzahl der Zwischenmuskelgräten weiter senken.

Wir erwarten jedoch das Auffinden von extremen Werten, die weit außerhalb der Normalverteilung liegen. Die Existenz solcher Mutanten kann auf Grund vieler Beispiele in der Biologie angenommen werden.

Bei der Suche nach alkaloidfreien Mutanten bei bitteren, alkaloidhaltigen Lupinenarten traten die erwarteten bitterstofffreien Formen in einer Häufigkeit von 1:20000 bis 1:1 Million auf. Beim Tabak der Sorte 'Havanna' konnte unter 20000 Individuen ein alkaloidfreies (= nikotinfreies) Individuum gefunden werden, und anthozyanfreie Exemplare fanden sich bei anthozyanhaltigen Landsorten des Spargels in einer Häufigkeit von 1:20000. Außer diesen Auslese-Arbeiten, die chemische Eigenschaften betreffen, sind in ähnlicher Weise physiologische Eigenschaften bearbeitet worden, wie z. B. die Auffindung parthenokarper Individuen bei Tomaten.

Gerade im Hinblick auf die Gräten scheint erwähnenswert, daß auch die Suche nach Formen mit extremen morphologischen Eigenschaften Erfolg haben kann. So konnte aus 10 Millionen Lupinen ein Exemplar mit nichtplatzenden Hülsen ausgelesen werden, eine Eigenschaft, die durch einen besonderen Bau bestimmter Sklerenchymfaserbündel bedingt ist. Zur Auffindung eines Champignons der Form 59c ohne Stiel, ohne Hut, ohne Lamellen und ohne Sporen wurden 10 Jahre benötigt.

Alle diese erwähnten Extremformen fanden sich weit außerhalb jeder Normalverteilung. Wenn man für das Vorhandensein von Zwischenmuskelgräten ein oder wenigstens wenige entscheidende Gene annimmt, muß man auch beim Karpfen das Auftreten grätenloser Formen erwarten, die dann weit außerhalb der Gauß-Kurve als Pik auftauchen.

Wir hoffen, da $B$ wir nach Auffinden grätenloser Exemplare durch planmäßige Paarung und Auslese in der Nachkommenschaft zur Fixierung der gewünschten Eigenschaft kommen können.

\section{Zusammenfassung}

Die Untersuchung von ca. 2000 Karpfen einer Population mit Hilfe von Röntgenaufnahmen ergab eine starke Streuung der Anzahl der Zwischenmuskelgräten. Bei einem Durchschnitt von ca. 100 Grätenspitzen pro Fisch traten als Extremwerte 70 und 134 Grätenspitzen pro Fisch auf. Es wurde festgestellt, daß die Anzahl der Grätenspitzen im Laufe der ersten 2 Lebensjahre zunimmt, wogegen die Anzahl der Grätenbasen sich nicht ändert. Auf Grund des Gesetzes der Parallelmutation wird das Auffinden extrem grätenarmer und grätenloser Formen erwartet.

\section{Literatur}

1: LIEDER, U.: Untersuchungsergebnisse über die Grätenzahlen bei 17 Süßwasser-Fischarten. Z. Fischeřei 10, 329-350 (1961). - 2. Meske, CH.: Karpfenaufzucht in Aquarien. Fischwirt 16, 309-316 (1966). - 3. MESKE, CH., Barbara LÜHR und W. Szablewskr: Fehlender Sexualrhythmus bei Karpfen in Warmwasserhaltung. Naturwissenschaften $54,291(1967)$. - 4. v. SENGBUSCH, R.: Fische ,ohne Gräten". Der Züchter 33, $284-286$ (1963). - 5. v. Sengbusch, R., Ch. Meske und W. SzABLEWSKI: Beschleunigtes Wachstum von Karpfen in Aquarien mit Hilfe biologischer Wasserklärung. Experientia 21, 614 (1965). - 6. v. SengBusch, R., Barbara LÜHR, CH. Meske und W. Szablewskr: Aufzucht voin Karpfenbrut in Aquarien. Archiv f. Fischereiwiss. 17, 89-94 (1966). - 7. V. Sengbusch, R., Ch. Meske, W. SZABLEWSKI und B. LüHR: Gewichtszunahme von Karpfen in Kleinstbehältern, zugleich ein Beitrag zur Aufklärung des. Raumfaktors. Z. Fischerei (1967 im Druck). - 8. WoyNÁrovich, E.: Ausreifen von Karpfenlaich in Zuger-Gläsern und Aufzucht der Jungfische bis zum Alter von 10 Tagen. Allg. Fischerei-Ztg. 86, 680 bis 682 (1961). 